\title{
Diagnostic value of far-IR water ice features in T Tauri disks
}

\author{
I. Kamp ${ }^{1}$, A. Scheepstra ${ }^{1}$, M. Min ${ }^{2}$, L. Klarmann ${ }^{2}$, and P. Riviere-Marichalar ${ }^{3}$ \\ ${ }^{1}$ Kapteyn Astronomical Institute, University of Groningen, Groningen, The Netherlands \\ e-mail: kamp@astro.rug.nl \\ 2 Anton Pannekoek Institute, University of Amsterdam, Amsterdam, The Netherlands \\ ${ }^{3}$ Instituto de Física Fundamental (CSIC), Calle Serrano 113b \& 123, 28006 Madrid, Spain
}

Received 27 November 2017 / Accepted 4 April 2018

\begin{abstract}
Aims. This paper investigates how the far-IR water ice features can be used to infer properties of disks around T Tauri stars and the water ice thermal history. We explore the power of future observations with SOFIA/HIRMES and SPICA's proposed far-IR instrument SAFARI.

Methods. A series of detailed radiative transfer disk models around a representative $\mathrm{T}$ Tauri star are used to investigate how the far-IR water ice features at 45 and $63 \mu \mathrm{m}$ change with key disk properties: disk size, grain sizes, disk dust mass, dust settling, and ice thickness. In addition, a series of models is devised to calculate the water ice emission features from warmup, direct deposit, and cooldown scenarios of the water ice in disks.

Results. Photodesorption from icy grains in disk surfaces weakens the mid-IR water ice features by factors of 4-5. The far-IR water ice emission features originate from small grains at the surface snow line in disks at distance of 10-100 au. Unless this reservoir is missing in disks (e.g., transitional disks with large cavities), the feature strength does not change. Grains larger than $10 \mu \mathrm{m}$ do not contribute to the features. Grain settling (using turbulent description) affects the strength of the ice features by at most $15 \%$. The strength of the ice feature scales with the disk dust mass and water ice fraction on the grains, but saturates for dust masses higher than $10^{-4} M_{\odot}$ and for ice mantles that increase the dust mass by more than $50 \%$. The various thermal histories of water ice leave an imprint on the shape of the features (crystalline and/or amorphous) and on the peak strength and position of the $45 \mu \mathrm{m}$ feature. SOFIA/HIRMES can only detect crystalline ice features that are much stronger than those simulated in our standard T Tauri disk model in deep exposures $(1 \mathrm{~h})$. SPICA/SAFARI can detect the typical ice features in our standard T Tauri disk model in short exposures (10 min).

Conclusions. The sensitivity of SPICA/SAFARI will allow the detailed study of the 45 and $63 \mu \mathrm{m}$ water ice feature in unbiased surveys of T Tauri stars in nearby star forming regions and an estimate of the mass of their ice reservoir. The water ice emission features carry an imprint of the thermal history of the ice, and thus can distinguish between various formation and transport scenarios. Amorphous ice at $45 \mu \mathrm{m}$ that has a much broader and flatter peak could be detected in deep surveys if the underlying continuum can be well characterized and the baseline stability of SAFARI is better than a few percent.
\end{abstract}

Key words. stars: low-mass - stars: pre-main sequence - infrared: planetary systems - protoplanetary disks

\section{Introduction}

During planet formation, ice plays a key role in multiple ways. Water ice enhances the solid mass reservoir available for planet formation by improving the sticking properties of small dust grains for coagulation into larger bodies (e.g., Wada et al. 2007, 2013; Wettlaufer 2010; Gundlach \& Blum 2015). More indirectly, ices are an extra opacity source, and thus affect the disk thermal structure. In addition, they could play an important role in providing the reservoir to make larger organic molecules (see review by Munoz Caro \& Dartois 2013).

In Herbig disks, water ice was first detected by Malfait et al. (1999) in the ISO/SWS and LWS spectra of HD 142527, a detection followed up later by Herschel/PACS (Min et al. 2016a). The disk contains an outer reservoir of highly crystalline ice (beyond $\sim 130 \mathrm{au}$ ), which comprises a major fraction of the available oxygen $(\sim 80 \%)$. Chiang et al. (2001) report the detection of the $45 \mu \mathrm{m}$ water ice feature in ISO/LWS spectra of CQ Tau and AA Tau; the spectra unfortunately lack full wavelength coverage of this feature. Later, Herschel/PACS spectra of a small sample of $\mathrm{T}$ Tauri stars also suffer from partial coverage of the ice features and baseline stability issues. However, McClure et al.
(2012) were able to report a detection of the $63 \mu \mathrm{m}$ crystalline ice feature in GQ Lup and McClure et al. (2015) in Haro 6-13 and DO Tau.

The history and evolution of the ices in disks remains unclear. Visser et al. (2009) show from cloud collapse simulations that water ice is incorporated into the outer reservoir of a protoplanetary disk without desorption (never heated above the sublimation temperature). Recent observations of the comet 67P/Churyumov-Gerasimenko with the Rosetta mission show high abundances of the noble gas argon; this suggests that the ice of that comet has never been heated above $\sim 20 \mathrm{~K}$ (Balsiger et al. 2015). On the other hand, the high fraction of cold crystalline ice seen in the disk around HD 142527 requires some heating and/or mixing processes within the disk.

Photodesorption processes can remove ices from grains in the surface layers of cold outer disks (Dominik et al. 2005; Woitke et al. 2009), thereby creating a cold water vapor reservoir. This cold water vapor was subsequently detected by Herschel/HIFI in the disks around TW Hya and DG Tau (Hogerheijde et al. 2011; Podio et al. 2013). After establishing the relevance of nonthermal desorption, Min et al. (2016a) model for the first time the water ice reservoir self-consistently in the disk around a Herbig star with the 2D thermal disk structure 
and photodesorption. They use an ice line description that is calibrated on thermo-chemical disk models.

In this work, we expand the disk modeling to T Tauri disks. We use the reference T Tauri disk model presented in Woitke et al. (2016; Sect. 2). By varying key disk parameters such as the disk size, dust mass, grain sizes, dust settling, and ice thickness, we exploit the diagnostic value of the far-IR water ice features at 45 and $63 \mu \mathrm{m}$ (Sect. 3). In addition, we explore whether we can observationally distinguish the three scenarios of warm-up, in situ formation, and cooldown for water ice. In Sect. 4, we discuss our results in the context of the approved SOFIA/HIRMES instrument and the proposed SPICA/SAFARI instrument.

\section{Disk models}

All disk models are calculated using the 3D Monte Carlo radiative transfer code MCMax (Min et al. 2009). The code uses a grain size distribution where the fraction of ices is a free parameter. We use the DIANA ${ }^{1}$ grain opacities (Min et al. 2016b), which were constructed to simultaneously fit observed thermal and scattered light properties in protoplanetary disks. The ice opacities are taken from Smith et al. (1994), and the amorphous ice opacities from Li \& Greenberg (1998); the code can use either opacities at a fixed temperature or temperature dependent opacities. In the following, we use $2 \mathrm{D}$ disk models and solve the continuum radiative transfer iteratively together with the location of the water-ice reservoir. For the latter, we use the formalism developed in Min et al. (2016a), where the snow line depends on the temperature, pressure, and UV radiation field (thermal and nonthermal desorption processes); the oxygen abundance is constrained by setting the total ice fraction in the disk $f_{\text {ice. }}$. Typically, three iterations are needed for the disk structure and spectral energy distribution (SED) to converge.

Table 1 summarizes the key parameters of the reference $\mathrm{T}$ Tauri disk model from Woitke et al. (2016). We focus here on passive irradiated disks where viscous heating plays a negligible role in the midplane close to the star. Since we investigate in this work, the far-IR water ice features typically originating from beyond $10 \mathrm{au}$, the precise radial position of the midplane snow line is not expected to affect our results.

The fraction of ice is fixed to 0.8 for the reference model and the disk parameter series including the thermal history of ice series. However, we include one model series where we explore the impact of the water ice fraction on the SED and the strength of the water ice features. The total dust mass is spread over silicates, carbon, and vacuum $(60 \%, 15 \%$, and $25 \%$, respectively). The fraction of ice is defined as

$M_{\text {ice }}=f_{\text {ice }} M_{\text {dust }}$.

In this way, changing the ice fraction does not change the underlying bare grain opacities. Having a nonzero ice fraction adds a mantle around each grain and the total mass of extra ice is spread using the underlying bare grain size distribution. The values of the first, second, and third moment of the bare grain size distribution are $\langle a\rangle=8.33 \times 10^{-2} \mu \mathrm{m},\left\langle a^{2}\right\rangle=1.245 \times 10^{-2} \mu \mathrm{m}^{2}$, and $\left\langle a^{3}\right\rangle=1.525 \times 10^{-1} \mu \mathrm{m}^{3}$. The ice fractions chosen here (see Table 2) explore a reasonable range since thermo-chemical disk models suggest that the grains increase in size by almost a factor of two due to the ice mantles (Chaparro-Molano \& Kamp

1 DIANA is the EU FP7 project "Disc Analysis" (PI: P. Woitke) that developed tools for the interpretation of multi-wavelengths observations of protoplanetary disks and applied them to a large number of protoplanetary disks using a consistent approach.
Table 1. Basic model parameters for the reference $\mathrm{T}$ Tauri disk.

\begin{tabular}{lll}
\hline \hline Quantity & Symbol & Value \\
\hline Stellar mass & $M_{*}$ & $0.7 M_{\odot}$ \\
Effective temperature & $T_{\text {eff }}$ & $4000 \mathrm{~K}$ \\
Stellar luminosity & $L_{*}$ & $1.0 L_{\odot}$ \\
Far-UV excess & $f_{\mathrm{UV}}$ & 0.01 \\
& $p_{\mathrm{UV}}$ & 1.3 \\
\hline Disk dust mass ${ }^{1}$ & $M_{\text {dust }}$ & $3.3 \times 10^{-4} M_{\odot}$ \\
Inner disk radius & $R_{\text {in }}$ & $0.07 \mathrm{au}$ \\
Outer disk radius & $R_{\text {out }}$ & $700 \mathrm{au}$ \\
Tapered edge radius & $R_{\text {taper }}$ & $100 \mathrm{au}$ \\
Column density power index & $\epsilon$ & 1.0 \\
Reference radius & $R_{0}$ & $100 \mathrm{au}$ \\
Scale height at $R_{0}$ & $H_{0}$ & $10.0 \mathrm{au}$ \\
Disk flaring power index & $\beta$ & 1.15 \\
\hline Minimum dust particle radius & $a_{\text {min }}$ & $0.05 \mu \mathrm{m}$ \\
Maximum dust particle radius & $a_{\text {max }}$ & $3000.0 \mu \mathrm{m}$ \\
Dust size dist. power index & $a_{\text {pow }}$ & 3.5 \\
Turbulent mixing parameter & $\alpha_{\text {settle }}$ & $10^{-2}$ \\
Max. hollow volume ratio & $V_{\text {hollow }}^{\text {max }}$ & $80 \%$ \\
Dust composition & $\mathrm{Mg}_{0.7} \mathrm{Fe}_{0.3} \mathrm{SiO}_{3}$ & $60 \%$ \\
(volume fractions) & amorph. carbon & $15 \%$ \\
& porosity & $25 \%$ \\
Grain material density & $\rho_{\text {grain }}$ & $2.076 \mathrm{~g} \mathrm{~cm}{ }^{-3}$ \\
\hline
\end{tabular}

Notes. ${ }^{(1)}$ The disk mass is a factor 3.3 higher than in the original Woitke et al. (2016) model. ${ }^{(2)}$ The outer radius is defined as the radius where the surface density column drops to $N_{\langle\mathrm{H}\rangle, \text { ver }}=10^{20} \mathrm{~cm}^{-2}$.

2016). The fractions of $0.4,0.8,1.2,1.6$, and 2.0 correspond to a grain size increase of a factor 1.9, 2.2, 2.4, 2.5, and 2.6, respectively, assuming an average water ice density of $1 \mathrm{~g} \mathrm{~cm}^{-3}$.

\subsection{Model series}

In order to isolate disk effects on the SED and the 45 and $63 \mu \mathrm{m}$ water ice features, the base choice for opacities is crystalline ice at $140 \mathrm{~K}$ unless stated otherwise. For comparison, the reference disk model is also calculated using amorphous ice opacities and without taking photodesorption into account. For all model series, only one of the parameters of the disk is altered at a time. This allows us again to isolate the effect of several properties of the disk on the water ice features. Table 2 shows all the parameters that were varied; the values of the reference disk model are found in Table 1.

An additional series was performed where the composition of the water ice is varied, based on its thermal history. We used the same series that are described by Smith et al. (1994):

- Series 1: warm-up from 10 to $150 \mathrm{~K}$.

- Series 2: direct deposit between 10 and $150 \mathrm{~K}$.

- Series 3: cooldown from 140 to $10 \mathrm{~K}$.

The warmup series refers to a scenario where the water ice is formed in cold, optically thick regions. After its formation it moves to warmer areas in the disk. We mimic this by using for each temperature interval in the disk the corresponding ice opacity from the warmup series of Smith et al. (1994). The water ice in the direct deposit scenario is formed locally and stays in the same thermal region. Also here, we change the ice opacity as a function of disk temperature, using this time the direct 
Table 2. Varied parameters for the disk models.

\begin{tabular}{lll}
\hline \hline Quantity & Symbol & Values \\
\hline Inner radius & $R_{\text {in }}$ & $0.07,0.2,1,10,30,50 \mathrm{au}$ \\
Tapering-off radius & $R_{\text {taper }}$ & $50,100,200 \mathrm{au}$ \\
Disk dust mass & $M_{\text {dust }}$ & $10^{-3}, 5 \times 10^{-4}, 3.3 \times 10^{-4}$, \\
& & $2 \times 10^{-4}, 10^{-4}, 10^{-5}, 10^{-6}$, \\
& & $10^{-7} M_{\odot}$ \\
Minimum grain size & $a_{\text {min }}$ & $0.05,0.5,2,30,50 \mu \mathrm{m}$ \\
Turbulent mixing & $\alpha_{\text {settle }}$ & $10^{-2}, 10^{-3}, 10^{-4}$ \\
Fraction of water ice & $f_{\text {ice }}$ & $0.4,0.8,1.2,1.6,2.0$ \\
\hline
\end{tabular}

deposit series from Smith's work. In the cooldown scenario, the water ice is formed in warm areas close to the midplane snow line $(\sim 0.5 \mathrm{au}$, around $140 \mathrm{~K})$ and then moves outwards to colder areas in the disk. This is simulated using the same methodology as the other two series, but now with the cooldown opacity series.

For each disk model, we calculate the disk thermal structure, extent of the ice reservoir, and spectral energy distribution self-consistently. In the following, we describe the analysis of the strength of the water ice features.

\subsection{Analysis of water ice features}

The trend that we see in the shape of the water ice features between our different disk models is quantified by using a polynomial best fit to the continuum of the SED. Given the strong changes in SED shape related to disk geometry, the placement of the continuum can be very uncertain. Also, observers have no way to choose a more refined method since the underlying continuum could not be estimated independently as we could do in the models. To avoid these problems, we chose the simplest solution, a linear fit between 40.0 and $57.5 \mu \mathrm{m}$ that covers the $45 \mu \mathrm{m}$ water ice feature. The $63 \mu \mathrm{m}$ feature cannot be analyzed this way because of the broad and weak nature of the feature and the uncertainty of the shape of the underlying continuum.

We define the height of the $45 \mu \mathrm{m}$ water ice features $S_{\lambda \text {,peak }}$ as

$S_{\lambda, \text { peak }}=\frac{F_{\lambda, \text { peak }}-F_{\lambda, \text { cont }}}{F_{\lambda, \text { cont }}}$,

where $F_{\lambda \text {,cont }}$ and $F_{\lambda \text {,peak }}$ are the continuum and peak flux of the $\mathrm{SED}$, respectively. Since the height of the features is sensitive to the definition of the continuum, trends are only considered real if they are significantly larger than the error bars. We estimate the errors by also using higher degree polynomials to fit the continuum, and derive a peak strength. Due to the specific shape of the continuum over the 30-60 $\mu \mathrm{m}$ wavelength range (mostly concave), the polynomials systematically provide higher peak values (typically 10-25\%) compared to the linear fit. The uncertainty within the polynomials is smaller, typically only $10 \%$.

\section{Results}

\subsection{Ice opacities and photodesorption}

Photodesorption is extremely important for the strength of the water ice features. Not taking this effect into account will overestimate their strengths by a factor of $4-5$ (Fig. 1). Also,

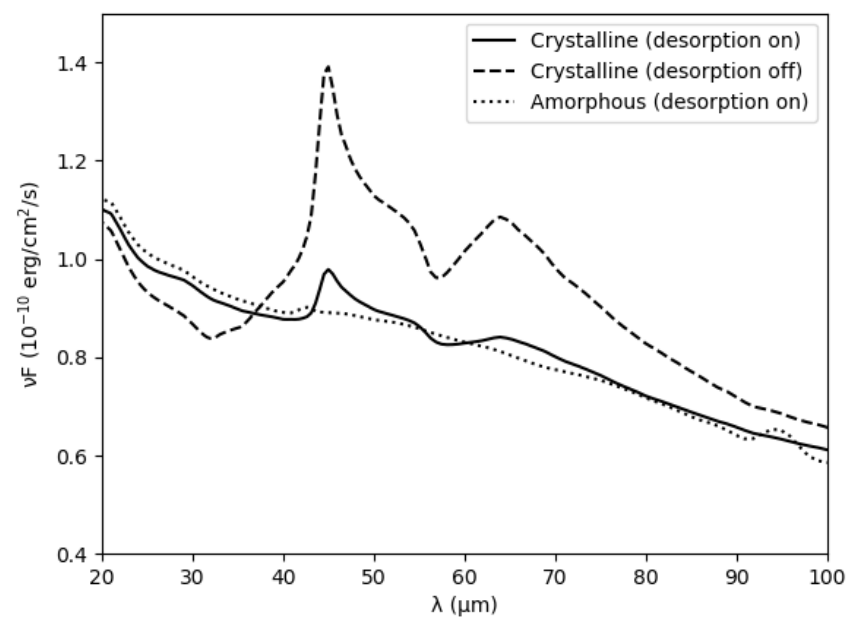

Fig. 1. SED of the reference disk model using crystalline ice opacities $(140 \mathrm{~K})$ with photodesorption switched on (solid line) and off (dashed line), and amorphous ice with photodesorption on (dotted line).

amorphous water ice produces features that are a factor of $\sim 7$ weaker than those of crystalline ice, to the extent that the peak strength for the $45 \mu \mathrm{m}$ feature becomes only 0.02 . Hence, in all subsequent parameter studies except for the "thermal history" series, we adopt crystalline ice opacities ( $140 \mathrm{~K})$. We note that this does not imply that we think water ice in disks has a temperature of $140 \mathrm{~K}$, but it merely ensures a straightforward and clean analysis of the features. The more realistic thermal history water ice scenarios are discussed in Sect. 3.5. It is also important to note that the underlying shape of the continuum and its absolute value change depending on the ice opacity choice. This is due to the self-consistent solution of the dust radiative transfer.

\subsection{Location of the water ice reservoir}

In the following we focus on the reference disk model to explain the location of the water ice reservoir and to illustrate where the 45 and $63 \mu \mathrm{m}$ ice features originate with respect to the continuum optical depth at those wavelengths.

Figure 2 shows the 2D temperature disk structure of the reference disk model. The thick white, dashed contour outlines the water ice reservoir of the disk (snow line). We note that at the outer radius of the disk there is no water ice present even though these regions are very cold. This is due to the interstellar radiation field, which causes photodesorption of ice mantles on dust grains in low density regions. The snow line is defined by the equation derived by Min et al. (2016a), an equilibrium between the vapor pressure and thermal/nonthermal desorption processes. The black lines show the region where the temperature is between 100 and $160 \mathrm{~K}$. This is roughly the area where the crystallization of water ice is happening (Smith et al. 1994). The red and yellow lines show the location where the photons can escape the disk without further absorption and re-emission. Photons emitted below the vertical optical depth $\tau=1$ line at 45 and $63 \mu \mathrm{m}$ will get absorbed again, and thus observers cannot obtain information about the structure of the disk below this region. The green and orange lines show where stellar photons with a wavelength of 0.55 and $3 \mu \mathrm{m}$, respectively, first interact with the disk. The stellar emission peaks at roughly $0.55 \mu \mathrm{m}$. Stellar photons with a wavelength of $3 \mu \mathrm{m}$ are not directly absorbed by the ice reservoir if the region has a hard cutoff and therefore the $3 \mu \mathrm{m}$ water ice feature may not show in observations of protoplanetary 


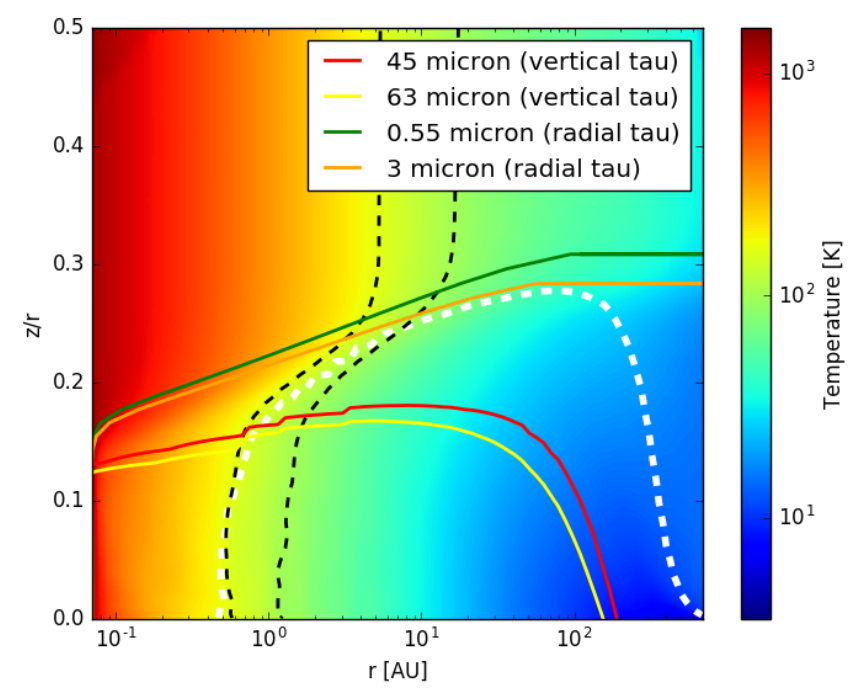

Fig. 2. Two-dimensional temperature disk structure for the reference disk model. The white dashed contour outlines the water ice reservoir. The black dashed lines are the 160 and $100 \mathrm{~K}$ temperature contours. The red and yellow contours denote the vertical optical depth $\tau=1$ at 45 and $63 \mu \mathrm{m}$. The green and orange contours denote the radial optical depth $\tau=1$ at 0.55 and $3 \mu \mathrm{m}$.

disks with simple standard geometries (full disk, no gaps, holes, no strong settling).

The 45 and $63 \mu \mathrm{m}$ water ice features in the SED of protoplanetary disks, originate between the snow line and the vertical optical depth $\tau=1$ lines at 45 and $63 \mu \mathrm{m}$. The following sections will use various model series to present in more detail the radial range over which the ice feature originate.

\subsection{Peak strength of the $45 \mu \mathrm{m}$ feature}

Table 3 shows the height of the $45 \mu \mathrm{m}$ water ice feature $S_{45 \text {,peak }}$ for the different disk model series. It is important to remember that the peak strength carries an error of typically less than $10 \%$ (via different polynomial methods).

In the following sections, we discuss in detail the different parameter series in two groups, parameters that do not change the water ice features and those that do. This enables us to assess the key science that can be learned from studying these features in the future.

\subsection{Disk properties that do not change the ice features}

The disk inner and outer radius, within the limits we probe here, do not affect the water ice features. However, they are very instructive in narrowing down the region where the ice features predominantly form. The peak height of the $45 \mu \mathrm{m}$ feature is constant between 0.11 and 0.14 throughout this series, that is they stay within $\pm 17 \%$, which is close to the error margin on the continuum placement $(10 \%)$.

Figure 3 shows that, while the continuum changes strongly with increasing disk inner radius from 0.07 to $50 \mathrm{au}$, the water ice features remain. This indicates that the dominant contribution to the water ice features comes from beyond $50 \mathrm{au}$. This is also illustrated by Fig. A.1; the ice reservoir (outlined by dashed white thick contour) stays unchanged for radii beyond $\sim 50 \mathrm{au}$.

Figure 4 shows that while the continuum changes strongly with increasing tapering-off radius of the disk (50-200 au), the water ice features again remain very stable. Increasing the
Table 3. Peak strength of the $45 \mu \mathrm{m}$ water ice feature in the reference model and the various $\mathrm{T}$ Tauri disk model series.

\begin{tabular}{|c|c|c|}
\hline Model series & Value & $S_{45 \text {,peak }}$ \\
\hline Reference (crystalline) & - & 0.134 \\
\hline Reference (amorphous) & - & 0.020 \\
\hline \multirow{6}{*}{$R_{\text {in }}(\mathrm{au})$} & 0.07 & 0.133 \\
\hline & 0.2 & 0.138 \\
\hline & 1 & 0.108 \\
\hline & 10 & $*$ \\
\hline & 30 & $*$ \\
\hline & 50 & $*$ \\
\hline \multirow[t]{3}{*}{$R_{\text {taper }}(\mathrm{au})$} & 50 & 0.141 \\
\hline & 100 & 0.130 \\
\hline & 200 & 0.128 \\
\hline \multirow[t]{6}{*}{$a_{\min }(\mu \mathrm{m})$} & 0.05 & 0.131 \\
\hline & 0.5 & 0.132 \\
\hline & 2 & 0.111 \\
\hline & 10 & 0.043 \\
\hline & 30 & $8 \times 10^{-4}$ \\
\hline & 50 & 0.0 \\
\hline \multirow{8}{*}{$M_{\text {dust }}\left(M_{\odot}\right)$} & $10^{-3}$ & 0.138 \\
\hline & $5 \times 10^{-4}$ & 0.141 \\
\hline & $3.3 \times 10^{-4}$ & 0.132 \\
\hline & $2 \times 10^{-4}$ & 0.127 \\
\hline & $10^{-4}$ & 0.127 \\
\hline & $10^{-5}$ & 0.107 \\
\hline & $10^{-6}$ & 0.050 \\
\hline & $10^{-7}$ & 0.0 \\
\hline \multirow[t]{3}{*}{$\alpha_{\text {settle }}$} & $10^{-2}$ & 0.132 \\
\hline & $10^{-3}$ & 0.146 \\
\hline & $10^{-4}$ & 0.157 \\
\hline \multirow[t]{5}{*}{$\overline{f_{\text {ice }}}$} & 0.4 & 0.087 \\
\hline & 0.8 & 0.128 \\
\hline & 1.2 & 0.149 \\
\hline & 1.6 & 0.170 \\
\hline & 2.0 & 0.176 \\
\hline
\end{tabular}

Notes. ${ }^{(*)}$ Indicates that we cannot measure the strength of the ice feature accurately due to the peak of the SED falling into the range of the water ice emission features.

tapering-off radius spreads the disk material to larger radii; however, the material that lies farther out does not substantially increase the strength of the ice feature. This indicates that the dominant contribution to the water ice features comes from within $100 \mathrm{au}$. The dust temperature within the ice reservoir beyond 100 au drops well below $50 \mathrm{~K}$, and so the icy dust grains are not at the peak of their emissivity. Figure A.2 also shows that the ice reservoir touches the outer disk radius for large tapering-off radii. However, the ice column density from those outer regions is likely too small to significantly contribute to the ice feature.

We use the turbulent $\alpha$ to parametrize dust settling (Dubrulle et al. 1995). The lower the turbulence, the stronger the dust settling in the outer disk. In the settled case, the large grains mostly settle towards the midplane, while the smaller micronsized grains are less affected. These are the carriers of the far-IR ice features, as demonstrated in the minimum grain size parameter series (see Sect. 3.5). The water ice features change weakly 


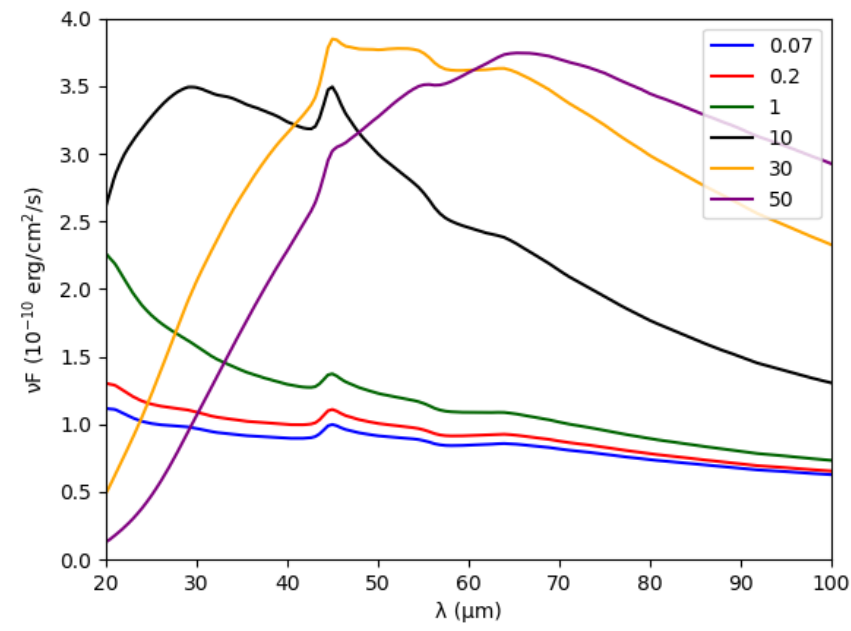

Fig. 3. SED for the disk models with disk inner radius varying between 0.07 and $50 \mathrm{au}$.

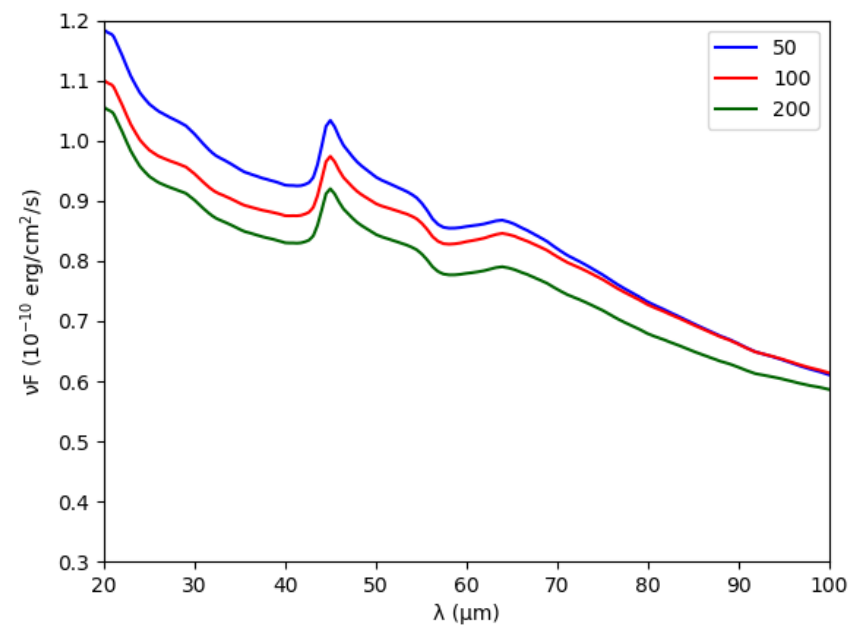

Fig. 4. SED for the disk models with disk tapering-off radius varying between 50 and $200 \mathrm{au}$.

with the level of turbulence (Fig. 5). The ice feature becomes $\sim 15 \%$ stronger for the lowest turbulence value compared with the highest value. This is largely due to the change in local continuum opacity pushing the $\tau \sim 1$ lines at 45 and $63 \mu \mathrm{m}$ closer to the disk midplane (see Fig. A.3).

Of course this result is limited by the intrinsic assumption of settling being an equilibrium process, and of ice evaporation/recondensation occurring on shorter timescales compared to possible vertical mixing processes.

\subsection{Dust/ice properties that change the ice features}

The 45 and $63 \mu \mathrm{m}$ water ice features are affected by the minimum grain size $a_{\mathrm{min}}$, the disk dust mass, the ice-to-refractory mass fraction (so the thickness of the ice mantle) and also the thermal history of the water ice. In the following, we summarize the key results from those four model series.

Figure 6 shows that while the absolute level of the continuum changes strongly with increasing minimum grain size, the water ice features remain very stable up to $a_{\text {min }} \sim 10 \mu \mathrm{m}$. Hence, we conclude that the dominant carrier of the far-IR water ice features are grains of sizes up to a few $\mu \mathrm{m}$. This also explains the result in the previous section that dust settling does not affect

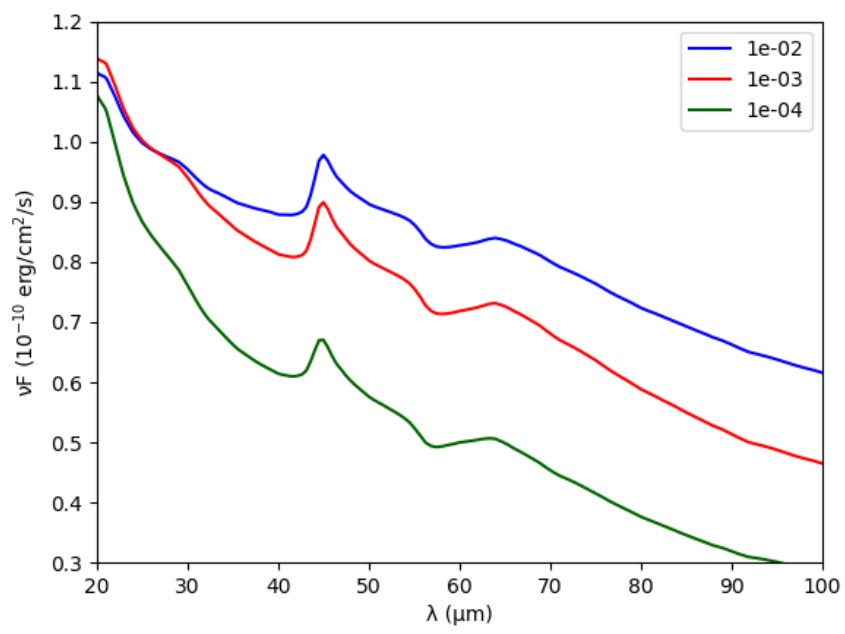

Fig. 5. SED for the disk models with different levels of turbulence $\alpha$ (see legend).

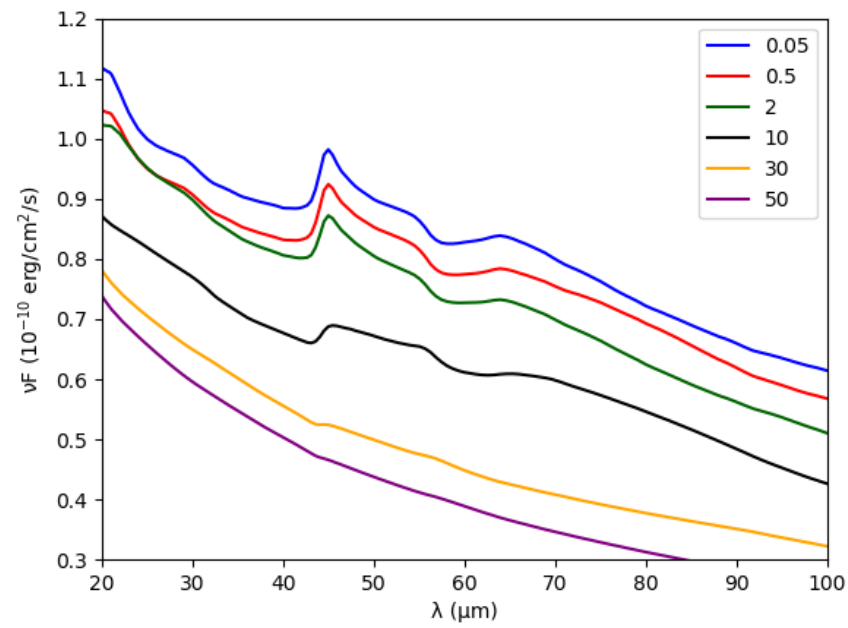

Fig. 6. SED for the disk models with minimum grain size varying between 0.05 and $50 \mu \mathrm{m}$ (see legend).

the strength of the 45 and $63 \mu \mathrm{m}$ water ice features. For a grain size distribution, where small grains are lacking, so $a_{\min }=10 \mu \mathrm{m}$, the $45 \mu \mathrm{m}$ feature becomes a factor of three smaller than the reference model $\left(a_{\min }=0.05 \mu \mathrm{m}\right)$.

The strength of the ice features correlates with dust mass until the disk reaches a mass of $\sim 10^{-4} M_{\odot}$ (Fig. 7). For higher dust masses, the ice features saturate and the feature strength levels off around 0.13 (Table 3 ). Typical dust masses of class II disks around stars with less than $1 M_{\odot}$ are below $2 \times 10^{-4} M_{\odot}$ (e.g., Pascucci et al. 2016), and hence it should be possible to estimate the total ice mass in many young star forming regions.

The ice fraction also produces a strong effect: the thicker the ice mantle, the greater the peak strength (Fig. 8). However, at high ice fractions of $>1.6$, the peak strength saturates. Adding more ice to the mantle does not contribute anything more to the emission; the ice becomes optically thick. Overall, an increase in the thickness of the ice mantle by a factor of 1.3 causes an increase in peak strength by a factor of approximately $2\left(f_{\text {ice }}=\right.$ 0.4 with respect to 1.6 ).

We would like to note here that, in the above model series we assume the ice to be in crystalline shape (i.e., formed at temperatures above $100 \mathrm{~K}$ ), causing prominent features. This assumption is plausible given the results from Min et al. (2016a). However, 


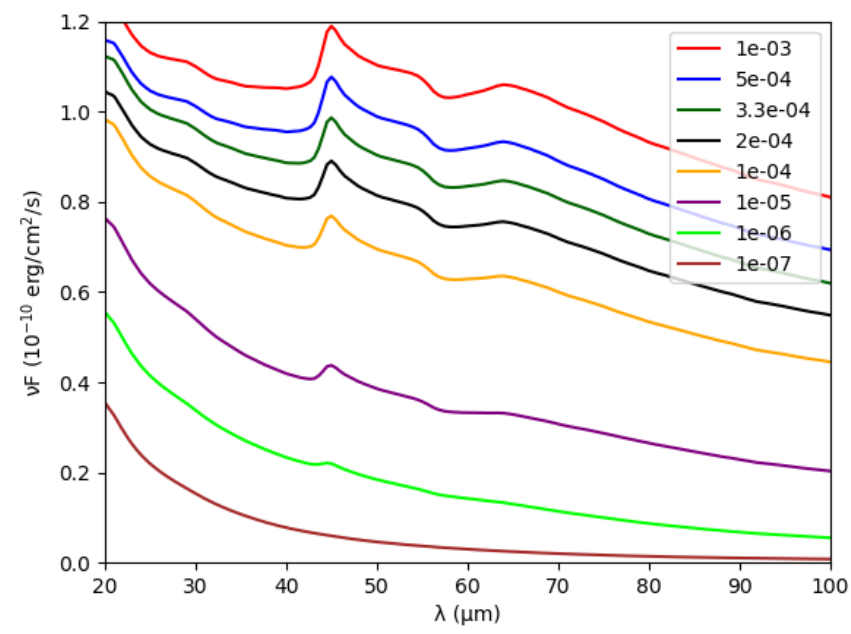

Fig. 7. SED for the disk models with disk dust masses varying between $10^{-3}$ and $10^{-7} M_{\odot}$ (see legend).

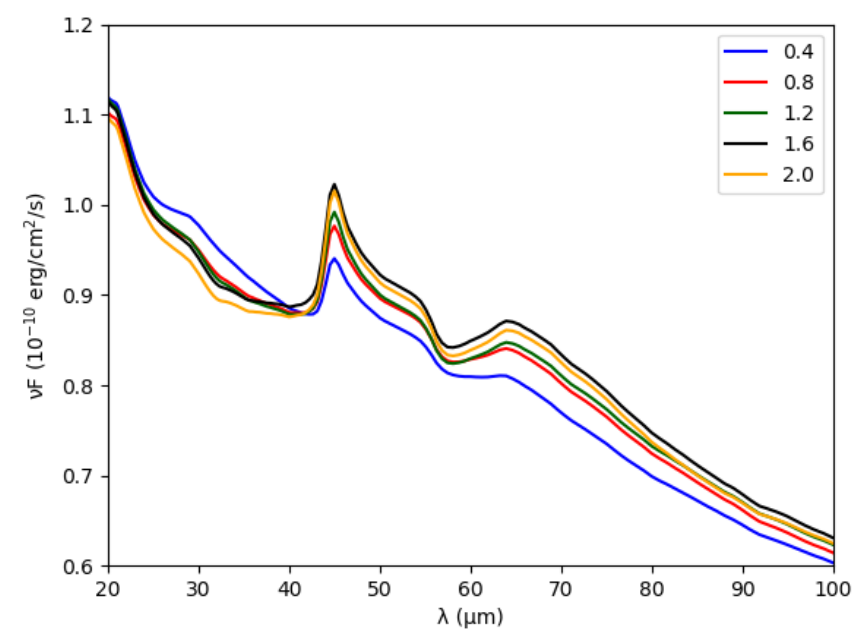

Fig. 8. SED for the disk models with different water ice fraction $f_{\text {ice }}$ on the grains $\left(f_{\text {ice }}=2\right.$ means that the models contains two times the dust mass added in ice mantles).

if the ice in the disk is formed and still located at temperatures below $100 \mathrm{~K}$, a significant fraction could be in amorphous form, which is more difficult to observe.

Figure 9 shows that, the different thermal histories of water ice will lead to clearly different feature strengths and shapes. Table 4 shows the peak wavelength of the two water ice features for these series. The $63 \mu \mathrm{m}$ feature shows no clearly defined peak for the warmup and direct deposit scenarios.

Interestingly, in the cooldown series we see a significant shift in the peak of the $45 \mu \mathrm{m}$ ice feature to shorter wavelengths $(43.55 \mu \mathrm{m})$ with respect to the reference series with opacities at $140 \mathrm{~K}(44.96 \mu \mathrm{m})$. This indicates that a large fraction of the ice feature originates instead from $\sim 70 \mathrm{~K}$ ice (see Table 1 in Smith et al. 1994). The $63 \mu \mathrm{m}$ feature also shows a shift corresponding to $\sim 90 \mathrm{~K}(62.6 \mu \mathrm{m})$. In the reference model, temperatures of $70-90 \mathrm{~K}$ correspond to temperatures at the snow line surface at a distance of 10-20 au.

In the direct deposit and warm-up series, the water ice features are more indicative of amorphous ice. Also, their peaks shift to longer wavelengths ( 45.93 and $46.42 \mu \mathrm{m}$ ) with respect to the reference model, again indicating, on average, low-ice temperatures in the emitting region $(<110 \mathrm{~K})$

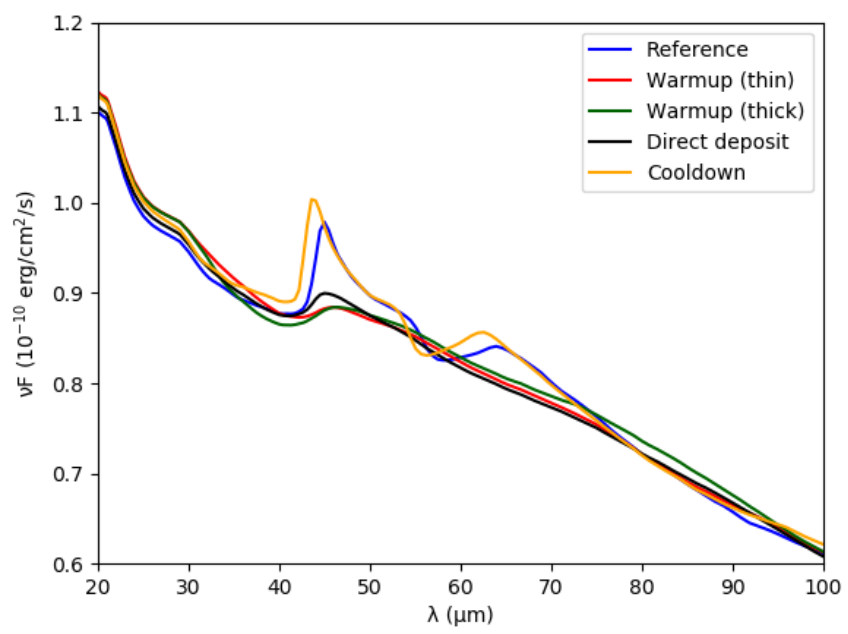

Fig. 9. SED for the disk models with different water ice thermal histories.

Table 4. Peak wavelengths position of the water ice features for the different thermal history scenarios.

\begin{tabular}{lll}
\hline \hline & $45 \mu \mathrm{m}$ & $63 \mu \mathrm{m}$ \\
\hline Reference & 44.96 & 63.91 \\
Warm-up (thin) & 45.93 & - \\
Warm-up (thick) & 46.42 & - \\
Direct deposit & 44.96 & - \\
Cooldown & 43.55 & 62.56 \\
\hline
\end{tabular}

in agreement with their amorphous nature and an origin beyond 6 au.

This model series is the only one where we include proper temperature dependent ice opacities. It shows that our approach to finding the spatial origin of these water ice features using the model series with varying inner and tapering-off radii might be too simple. For example, the emitting region of the ice features could change in the model series with increasing $R_{\text {in }}$, and an increasing surface area could compensate for the emission becoming weaker, thus leading to the apparent "constancy" of the far-IR water ice features over a wide range of disk geometries. The disk model series with water ice histories may provide here a more reliable estimate of the main emitting region through the measurement of the $45 \mu \mathrm{m}$ ice feature peak.

\section{Future observations}

\subsection{SOFIA/HIRMES}

HIRMES was selected as a third-generation instrument for the SOFIA Observatory. The SOFIA Observatory consists of a $2.5 \mathrm{~m}$ telescope mounted inside a modified Boeing 747SP operating at altitudes of $12-14 \mathrm{~km}$. HIRMES is an infrared spectrograph operating between 25 and $122 \mu \mathrm{m}$ with a spectral resolving power of $350-650$ in low resolution mode and $5 \times 10^{4}-10^{5}$ in high resolution mode. HIRMES will be commissioned on SOFIA in early to mid 2019. For the low resolution mode, rebinning to a resolution of 100 , the expected sensitivity is $\sim 0.04 \mathrm{Jy}$ ( $1 \sigma$ in $1 \mathrm{~h}$; Klaus Pontoppidan, priv. comm.).

Figure 10 shows the noise level that HIRMES will achieve in a $1 \mathrm{~h}$ exposure for our model series using $R=100$. Even though it could provide detections in the case of brighter sources and/or 


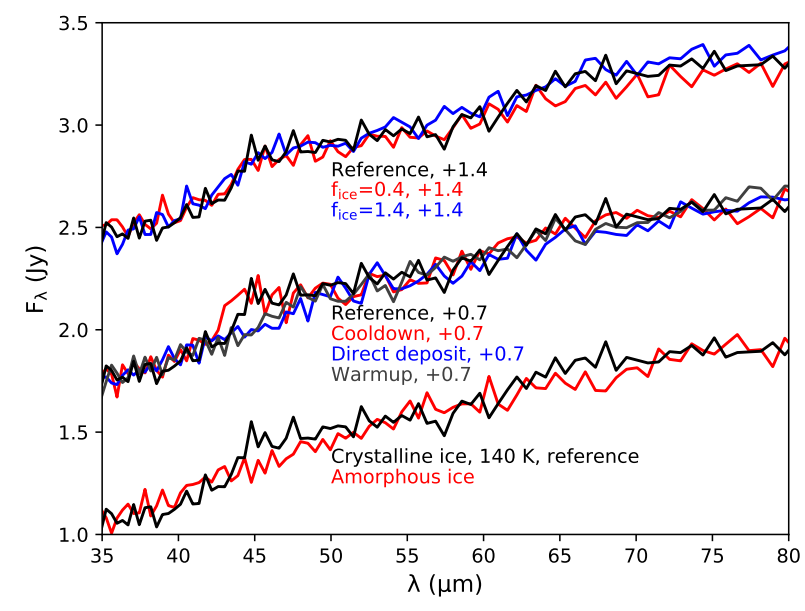

Fig. 10. Simulated HIRMES spectra for several ice model series: crystalline ice and amorphous ice, different water ice thermal histories shifted upwards by $0.7 \mathrm{Jy}(+0.7)$, and different ice thicknesses shifted upwards by $1.4 \mathrm{Jy}(+1.4)$. The exposure time is $1 \mathrm{~h}$ and the resolution is $R=100$.

for particularly strong features (e.g., very high ice fraction, crystalline ice at $140 \mathrm{~K}$ ), for most of the model simulations presented in this paper detailed studies of the ice properties and thermal history will be challenging with a resolution of $R=100$. Given the current sensitivities and our model predictions, ice studies of $\mathrm{T}$ Tauri disks with HIRMES will require long exposure times, for typical T Tauri continuum fluxes (1 Jy and lower) even beyond $1 \mathrm{~h}$.

\subsection{SPICA/SAFARI}

The SPace Infrared telescope for Cosmology and Astrophysics (SPICA) is a joint Japanese/European $2.5 \mathrm{~m}$ cooled telescope proposed for ESAs M5 call. The SAFARI instrument on board will allow spectroscopy between 34 and $230 \mu \mathrm{m}$ at a resolution of $300-11000$. In low resolution mode, it will reach $0.31,0.45$, and $0.72 \mathrm{mJy}(5 \sigma$ in $1 \mathrm{~h})$ in the $\mathrm{SW}(34-56 \mu \mathrm{m})$, MW $(54-89 \mu \mathrm{m})$, and LW $(87-143 \mu \mathrm{m})$ band, respectively.

Figure 11 shows the noise level that SAFARI will achieve in a short $10 \mathrm{~min}$ exposure for our model series using $R=300$. The SAFARI instrument with its high sensitivity will be able to distinguish between cooldown and direct deposit/warmup ice histories, but also ice thicknesses, i.e., ice fractions. The short exposure times $(10 \mathrm{~min})$ will enable ice surveys of hundreds of $\mathrm{T}$ Tauri disks with the SPICA mission. The most important limitation will be the baseline stability and the calibration of the instrument. For detailed ice characterization studies, we require a stability of better than a few percentage points across the $35-100 \mu \mathrm{m}$ spectral range.

Long exposures $(1 \mathrm{~h})$ can provide more reliable ice fraction measurements. However, the distinction between direct deposit and warmup scenarios, and the detection of amorphous ice will remain difficult and hinges mostly on a reliable continuum placement due to the broadness of the weak features.

\section{Discussion}

The constant nature of the far-IR water ice features against physical disk parameters such as disk sizes and settling makes these features a robust probe of the grain and ice history in disks.

A closely related water ice feature study is McClure et al. (2015). One of their Herschel observations, DO Tau, shows

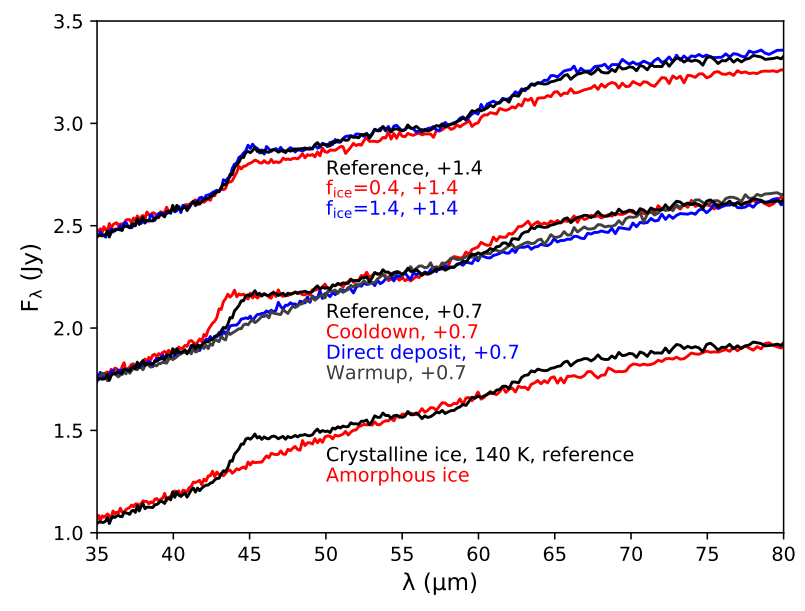

Fig. 11. Simulated SAFARI spectra for several ice model series: crystalline ice and amorphous ice, different water ice thermal histories shifted upwards by $0.7 \mathrm{Jy}(+0.7)$ and different ice thicknesses shifted upwards by $1.4 \mathrm{Jy}(+1.4)$. The exposure time is $10 \mathrm{~min}$ and the resolution is $R=300$.

far-IR continuum fluxes very close to our reference model. The peak strength of the detected feature at $63 \mu \mathrm{m}$ is $\sim 0.2$, at least a factor of two stronger than the feature we typically see using crystalline water ice in our reference model. The other water ice detections they report are even stronger. On the other hand, the weak features predicted from our "thermal history" modeling series would have been undetectable given the typical PACS noise level. The only way in our modeling approach to enhance the strength of the $63 \mu \mathrm{m}$ feature to a level that is detectable by PACS would be to neglect the photodesorption and have the water ice reservoir extend high up in the disk. It is however important to keep in mind that the continuum placement with PACS was very difficult due to the pointing stability and the data was obtained in range spectroscopy mode $(51-73 \mu \mathrm{m})$, thus only covering the central part of the feature without much neighboring continuum.

The thermal history modeling series illustrates the power of having access to the peak position of the $45 \mu \mathrm{m}$ ice feature, the strength of the peak, and the shape of the 45 and $63 \mu \mathrm{m}$ feature complex. If the ice is purely amorphous, the $63 \mu \mathrm{m}$ feature will be hard to distinguish from the continuum due to its very broad nature. However, the shorter wavelength feature remains in that case an important diagnostic, and the combination of peak wavelength and strength has the potential to discriminate between in situ formation and a scenario where the ice has been formed cold either in the precursor molecular cloud or in the outer disk and later distributed more widely through radial migration processes. The opposite process, a formation close to the midplane snow line and later migration outwards leaves the ice in a crystalline state, which is clearly detectable in both far-IR water ice features.

The next step should be to couple detailed grain growth/ migration models with the self-consistent snow line and dust radiative transfer approach. The optical depth of the disk will likely change if the grain size distribution varies with position in the disk. This could affect the appearance and strength of the water ice features. A step in this direction is the dust settling series in which the height of the far-IR continuum of the disk changes substantially. Similar changes can be expected for a varying radial distribution driven by dust migration. Once the smaller dust grains change their spatial distribution, the UV/optical $\tau \sim 1$ line will also change and this can also change the location of the snow line in the disk surface. Dust drift 
models suggest a high efficiency in depleting small grains ( $\mu$ m-sized) from a wide region around $\sim 80$ au (region IV in Fig. 2 of Birnstiel et al. 2015). This is the region we identify here as the main contributor to the water ice thermal emission feature. Leaving this region devoid of particle sizes that can produce the thermal ice emission features (grains $\lesssim 10 \mu \mathrm{m}$ ) would have an effect on the strength of the feature.

It is interesting to note that the absence of a water ice feature in the spectrum does not imply the absence of an extended water ice reservoir. This can be caused by the absence of grains smaller than $10 \mu \mathrm{m}$ in the outer parts of the disk ( $~ 230 \mathrm{au}$ ). The disk model with $a_{\min }=30 \mu \mathrm{m}$ shows no features in the SED, but still shows a significant ice reservoir.

\section{Conclusions}

This paper studies for the first time the far-IR water ice features in a self-consistent modeling approach to set the location of the water snow line together with consistent dust/ice opacities and dust disk thermal structure. We demonstrate that the photodesorption of water ice is key in the quantitative interpretation of the peak strengths of the 45 and $63 \mu \mathrm{m}$ features. The water ice features originate from grains smaller than $\sim 10 \mu \mathrm{m}$.

The water ice features originate predominantly at the surface snow line of the disk between 10 and $100 \mathrm{au}$, which means that they are very robust against several disk parameters such as inner and outer radii, the grain size distribution (within limits), and dust settling. However, the feature strength scales with disk dust mass in the range of typical T Tauri disks. The features also change substantially with the thickness of the ice mantle, although the strength levels off for mantles much thicker than 2.5 times the original grain sizes.

Most interestingly, the features do carry an imprint of the thermal history of the ice and thus can distinguish between a warmup scenario for the water ice (where water ice is formed in cold, optically thick regions and then moves to warmer areas in the disk) and a cooldown scenario (where water ice is formed in warm areas close to the midplane snow line and then moves outwards to colder areas in the disk). The direct deposit scenario (ice stays where it was formed locally in the disk) resembles qualitatively the warmup scenario and while the peak strength change of the $45 \mu \mathrm{m}$ feature may not allow a discrimination, the shift in the peak wavelengths to shorter values might be a discriminator.

Given the results of our ice model series, simulations using the sensitivities for the planned SOFIA/HIRMES instrument show that the low resolution and the sensitivity will lead to severely limited ice studies - beyond simple detection - in $\mathrm{T}$ Tauri disks at the distance of typical star forming regions. The $45 \mu \mathrm{m}$ ice feature is strong enough to be detected if it originates from warm grains with thick ice mantles that are crystalline in nature. Similar simulations for the SPICA/SAFARI instrument show that the differences in peak strengths and the wavelength shifts in the peak position can both be easily measured in samples of several hundred T Tauri disks (exposure times of $10 \mathrm{~min}$ ). At low spectral resolution $(R=300)$ weak flat ice features at $45 \mu \mathrm{m}$ (e.g., amorphous, direct deposit, warmup of ice) require a good continuum characterization in deep surveys.

Acknowledgements. IK and MM acknowledge funding from the EU FP7-2011 under Grant Agreement no. 284405. LK is supported by a grant from the Netherlands Research School for Astronomy (NOVA). PRM thanks ERC under ERC-2013-SyG, G.A. 610256 NANOCOSMOS. We thank the anonymous referee for the comments that improved the clarity of the paper and the suggestion to include the dust mass series.

\section{References}

Balsiger, H., Altwegg, K., Bar-Nun, A., et al. 2015, Sci. Adv., 1, e1500377 Birnstiel, T., Andrews, S. M., Pinilla, P., \& Kama, M. 2015, ApJ, 813, L14 Chaparro-Molano, G., \& Kamp, I. 2016, in Young Stars and Planets Near the Sun, eds. J. H. Kastner, B. Stelzer, \& S. A. Metchev, IAU Symp., 314, 189

Chiang, E. I., Joung, M. K., Creech-Eakman, M. J., et al. 2001, ApJ, 547, 1077 Dominik, C., Ceccarelli, C., Hollenbach, D., \& Kaufman, M. 2005, ApJ, 635, L85

Dubrulle, B., Morfill, G., \& Sterzik, M. 1995, Icarus, 114, 237

Gundlach, B., \& Blum, J. 2015, ApJ, 798, 34

Hogerheijde, M. R., Bergin, E. A., Brinch, C., et al. 2011, Science, 334, 338

Li, A., \& Greenberg, J. M. 1998, A\&A, 331, 291

Malfait, K., Waelkens, C., Bouwman, J., de Koter, A., \& Waters, L. B. F. M. 1999, A\&A, 345, 181

McClure, M. K., Manoj, P., Calvet, N., et al. 2012, ApJ, 759, L10

McClure, M. K., Espaillat, C., Calvet, N., et al. 2015, ApJ, 799, 162

Min, M., Dullemond, C. P., Dominik, C., de Koter, A., \& Hovenier, J. W. 2009, A\&A, 497, 155

Min, M., Bouwman, J., Dominik, C., et al. 2016a, A\&A, 593, A11

Min, M., Rab, C., Woitke, P., Dominik, C., \& Ménard, F. 2016b, A\&A, 585, A13

Munoz Caro, G. M., \& Dartois, E. 2013, Chem. Soc. Rev., 42, 2173

Pascucci, I., Testi, L., Herczeg, G. J., et al. 2016, ApJ, 831, 125

Podio, L., Kamp, I., Codella, C., et al. 2013, ApJ, 766, L5

Smith, R. G., Robinson, G., Hyland, A. R., \& Carpenter, G. L. 1994, MNRAS, 271,481

Visser, R., van Dishoeck, E. F., \& Black, J. H. 2009, A\&A, 503, 323

Wada, K., Tanaka, H., Suyama, T., Kimura, H., \& Yamamoto, T. 2007, ApJ, 661, 320

Wada, K., Tanaka, H., Okuzumi, S., et al. 2013, A\&A, 559, A62

Wettlaufer, J. S. 2010, ApJ, 719, 540

Woitke, P., Thi, W.-F., Kamp, I., \& Hogerheijde, M. R. 2009, A\&A, 501, L5

Woitke, P., Min, M., Pinte, C., et al. 2016, A\&A, 586, A103 


\section{Appendix A: SEDs and disk structures}

\section{A.1. Disk inner radius and tapering-off radius}

Figures A.1 and A.2 illustrate the temperature distribution in the disk models with varying inner radius and tapering-off radius. To assess where the water ice features originate, we also show the snow line and the vertical optical depth $\tau=1$ contours for 45 and $63 \mu \mathrm{m}$ and the radial optical depth $\tau=1$ at $0.55 \mu \mathrm{m}$.

\section{A.2. Dust settling}

Figure A.3 shows the 2D temperature distribution for disk models with various degrees of settling $\left(\alpha_{\text {turb }}=10^{-2}\right.$ to $\left.10^{-4}\right)$. The change in opacities is strongest in the outer disk, and hence temperature changes there are largest. Lower values for $\alpha_{\text {turb }}$ lead to strong dust settling towards the midplane, thus reducing the opacities in the upper/outer layers. This shifts the snow line to lower heights (from $\sim 0.3$ at 100 au for $\alpha_{\text {turb }}=10^{-2}$ to $\sim 0.25$ for $\alpha_{\text {turb }}=10^{-4}$ ) and produces a larger water vapor reservoir in the outer disk.

\section{A.3. Minimum grain size}

When we change the minimum grain size from 0.05 to $50 \mu \mathrm{m}$, the optical depth in the disk changes dramatically, as illustrated in Fig. A.4. Removing the smaller grains lowers the opacity and the greatest impact is seen in the surface layers, especially in the change of height at which the disk becomes optically thick (green contour, $\tau=1$ at $0.55 \mu \mathrm{m})$. Subsequently, the snow line also moves closer to the midplane ( $\sim 0.3$ at 100 au for $a_{\min }=0.05 \mu \mathrm{m}$ grains to $\sim 0.2$ for $a_{\min }=50 \mu \mathrm{m}$ grains).

If the disk surfaces are strongly depleted in small grains, there is an increasing chance of observing the ice feature also in scattered light; the green radial optical depth line touches the snow line, i.e., optical stellar light can be scattered off icy grains even at large distances from the star (out to several $10 \mathrm{au}$ ).

\section{A.4. Disk dust mass}

The disks become increasingly transparent at mid-IR wavelengths when the dust mass decreases from $10^{-3}$ to $10^{-7} M_{\odot}$ (see red and yellow contour in Fig. A.5). The full ice column density will be visible for dust masses below $10^{-4} M_{\odot}$ beyond $100 \mathrm{au}$ and for dust masses below $10^{-6} M_{\odot}$ beyond $2 \mathrm{au}$. At the same time, the disk ice reservoir (white dashed contours) shrinks because the disk becomes warmer with decreasing dust mass and photodesorption can act at lower disk height due to an overall lower optical depth to UV photons. Especially, in the lowest disk mass models, the change in opacity due to the presence of ice is now clearly visible in the optical depth contours.

\section{A.5. Ice fraction}

Figure A.6 clearly shows that the water ice fraction has a negligible impact on the disk thermal structure. It contributes very little to the overall grain energy balance. The explanation lies in the implementation, where the ice fraction determines an amount of ice to be added on top of the bare grain opacities. In that sense, changing the ice fraction does not change the underlying bare grain opacities. The grains become effectively thicker by growing more ice on the surface.

\section{A.6. Warmup versus cooldown scenario}

Figure A.7 shows the difference between using fixed temperature $140 \mathrm{~K}$ crystalline ice opacities in the reference model and the full temperature dependent ice opacities from the cooldown series. While the temperature changes in the disk model are very small, there is a shift in the optical depth at $45 \mu \mathrm{m}$ to lower depths in the cooldown model. It is clear that the crystalline fixed $140 \mathrm{~K}$ ice opacity of the reference disk model systematically overestimates the ice opacity. 

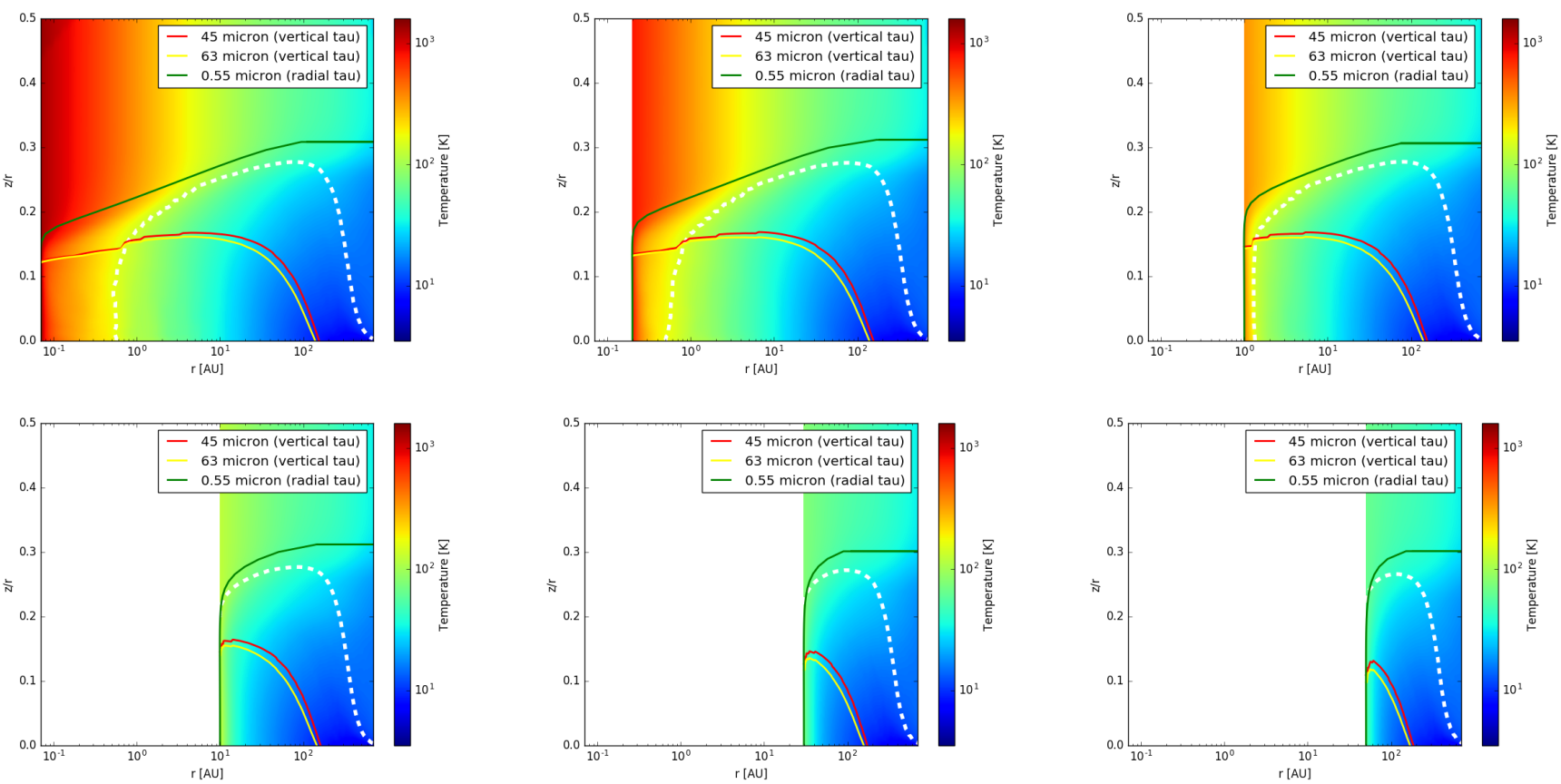

Fig. A.1. Two-dimensional (2D) dust temperature distribution in the model series where the disk inner radius changes from 0.07 to 50 au. The contour lines have the same meaning as in Fig. 2.
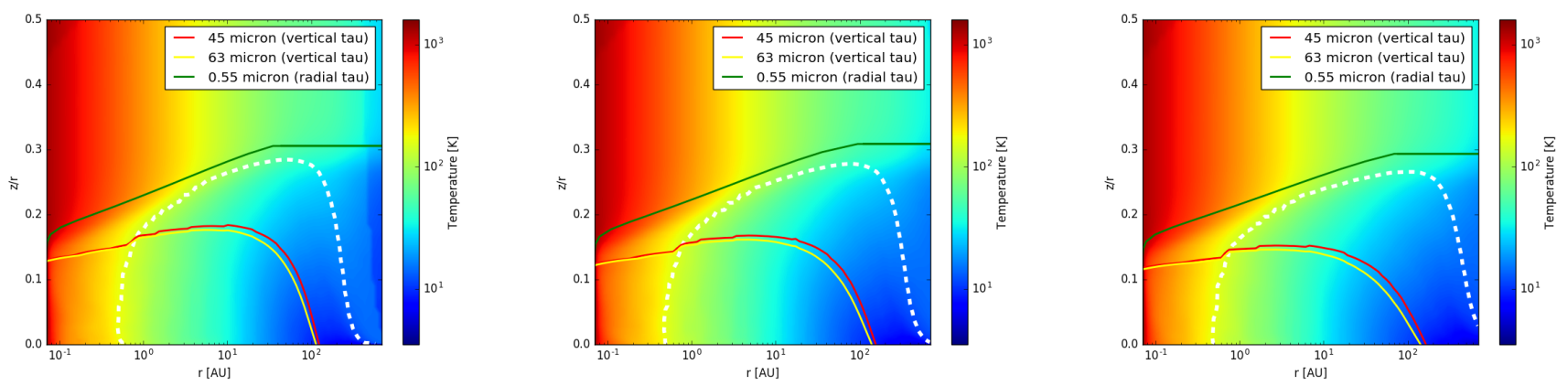

Fig. A.2. Two-dimensional (2D) dust temperature distribution in the model series where the disk tapering-off radius changes from 50 to 200 au. The contour lines have the same meaning as in Fig. 2.
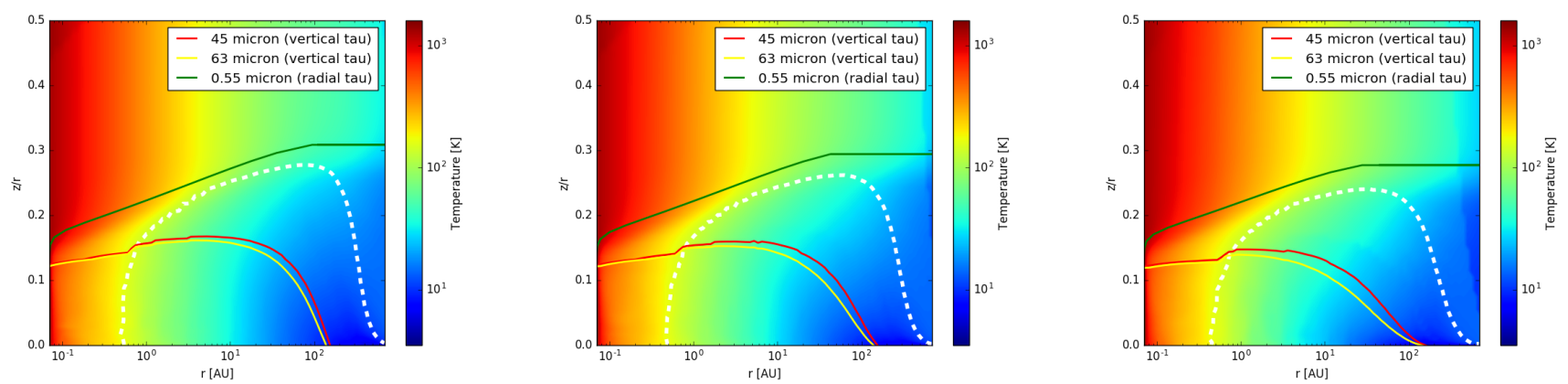

Fig. A.3. Two-dimensional (2D) dust temperature distribution in the model series where the turbulence decreases from 0.01 to $10^{-4}$. The contour lines have the same meaning as in Fig. 2. 
I. Kamp et al.: Diagnostic value of far-IR water ice features in T Tauri disks
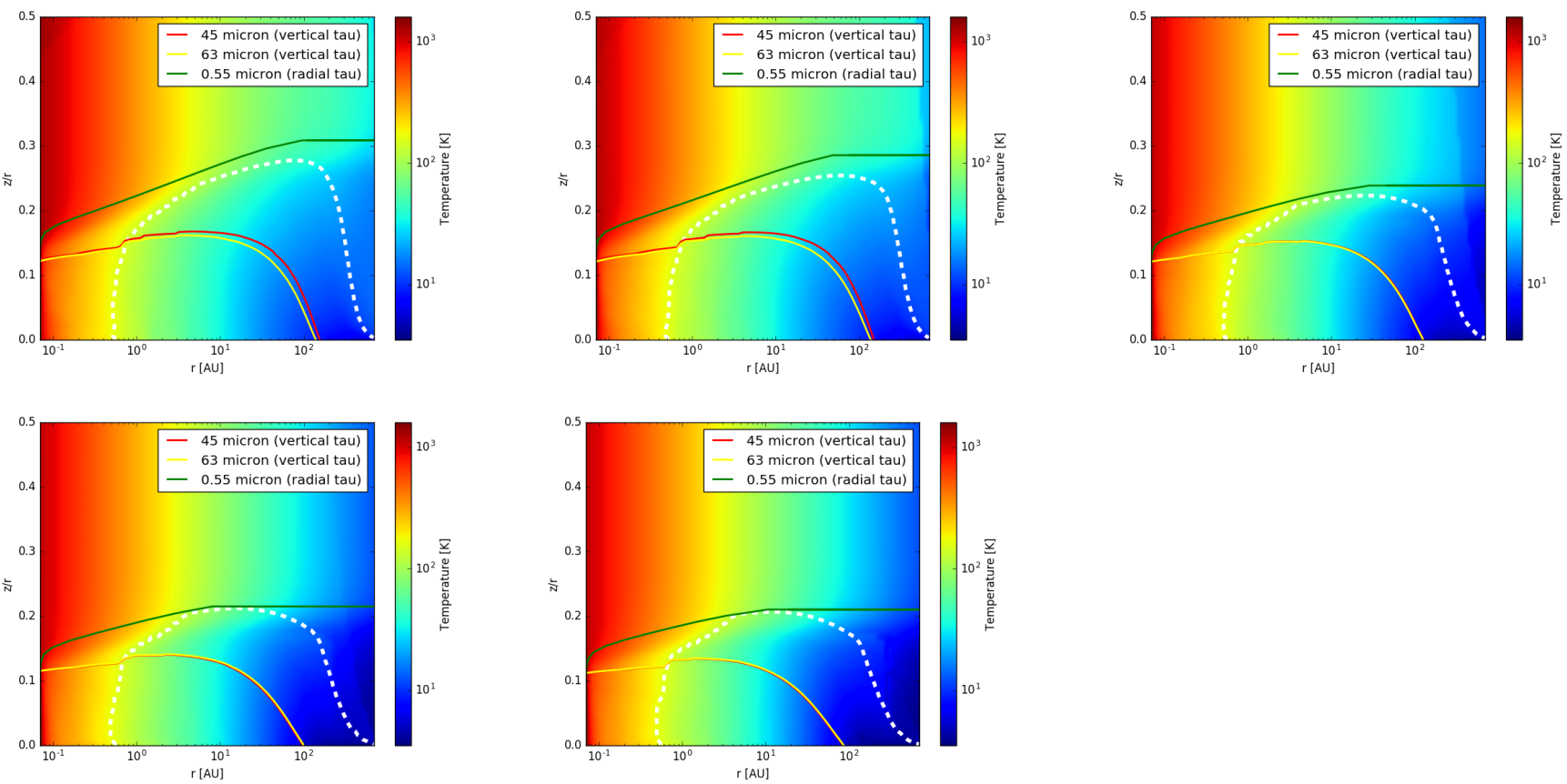

Fig. A.4. Two-dimensional (2D) dust temperature distribution in the model series where the minimum grain size increases from 0.05 to $50 \mu \mathrm{m}$. The contour lines have the same meaning as in Fig. 2.
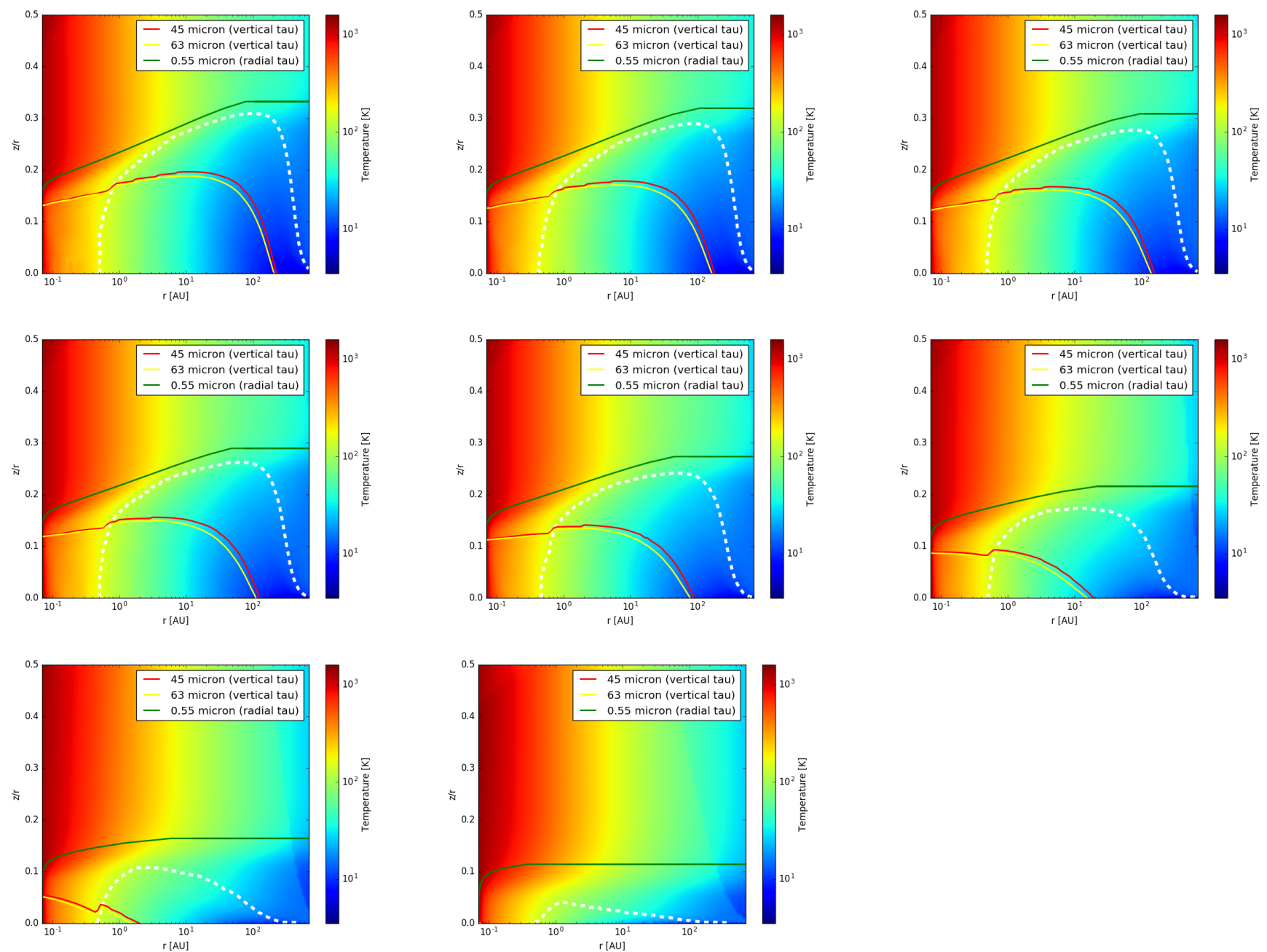

Fig. A.5. Two-dimensional (2D) dust temperature distribution in the model series where the disk dust mass decreases from $10^{-3}$ to $10^{-7} M_{\odot}$. The contour lines have the same meaning as in Fig. 2. 
A\&A 617, A1 (2018)
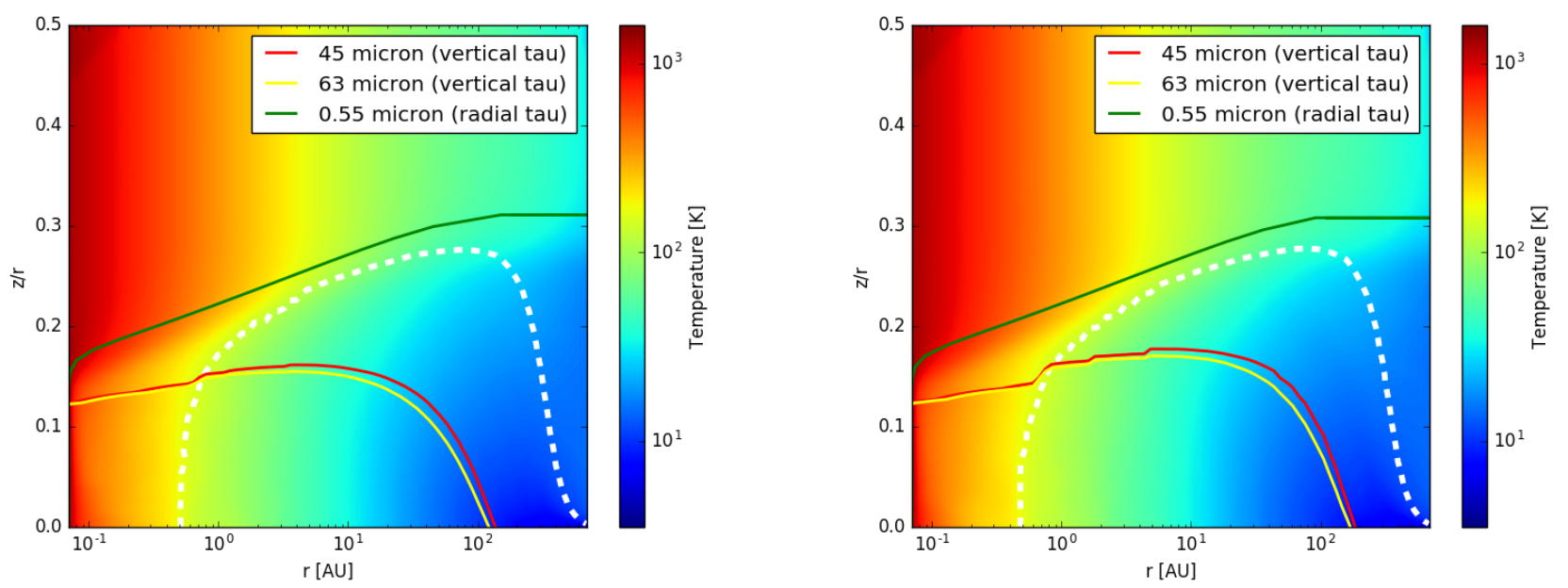

Fig. A.6. Two-dimensional (2D) dust temperature distribution in the model series with different water ice fraction of 0.1 and 2 . The contour lines have the same meaning as in Fig. 2.
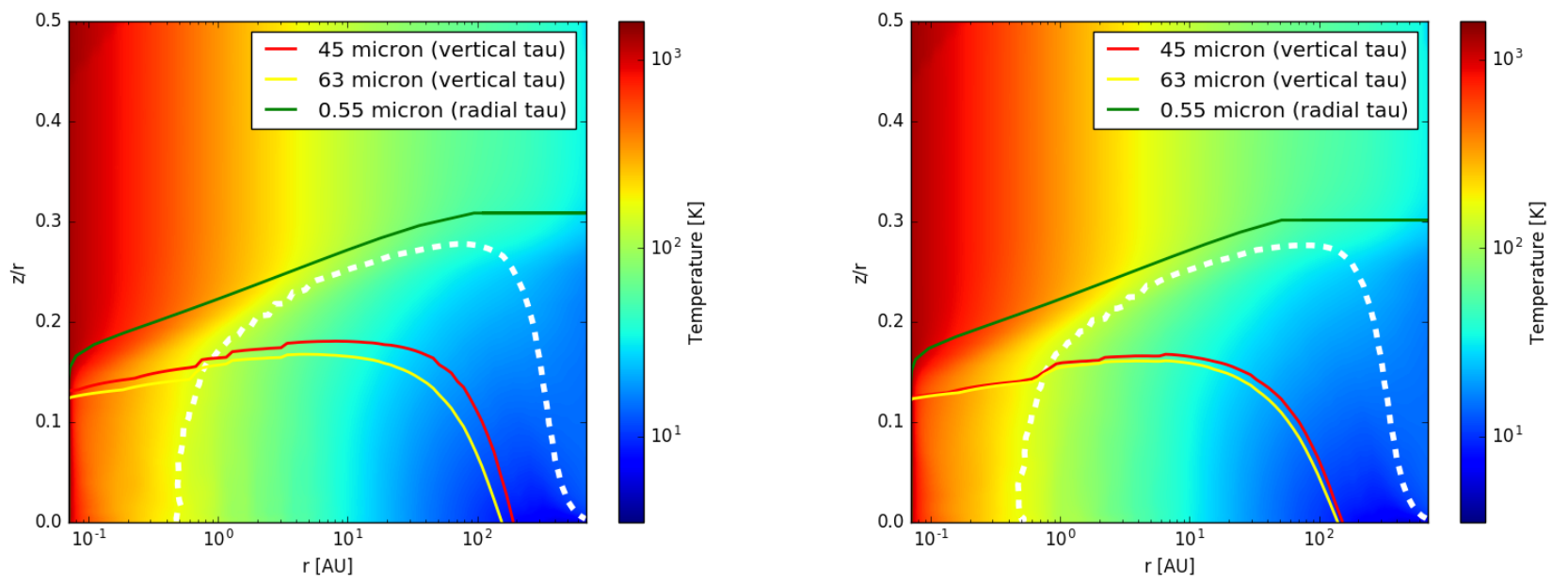

Fig. A.7. Two-dimensional (2D) dust temperature distribution in the model series with different water ice thermal histories; for comparison, the first panel shows the reference disk model. The contour lines have the same meaning as in Fig. 2. 